Quaderni di Geografia Cahiers de Gécongaty rataphie

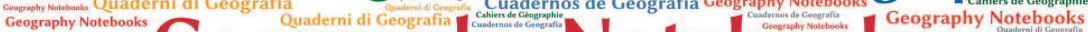
Cuadernos de Geografia Geography Notebooks $U 201000$ Cahiers de Géographi Cahiers de Géographie cahiers de Géographie Cuadernos de Geografía Cahiers de Géographie Cahiers de Géographie Cuadernos de Geografía Geography Notebooks

\author{
$4(2021)$ \\ 1 \\ Teatro di suoni. \\ Spazi acustici teatrali e territoriali
}

A cura di

Martino Mocchi, Lorena Rocca, Demis Quadri and Carlotta Sillano

EDITORIAL

Teatro di suoni per l'attaccamento ai luoghi. Uno sguardo geografico 11

Lorena Rocca

Per un teatro di suoni. Riflessioni su possibili dimensioni sonore nelle 23 creazioni site-specific di physical theatre

Demis Quadri

INTRODUCTION

Teatro di suoni. Spazi acustici teatrali e territoriali

Demis Quadri e Lorena Rocca

SPECIAL Issue

I suoni di Mantova come strumenti di interpretazione del paesaggio.

Tra turismo sostenibile ed educazione al patrimonio culturale

Valeria Pecorelli, Franca Zuccoli, Alessandra De Nicola, Enrico Squarcina

Il paesaggio sonoro campano tra contemporaneità e nuove forme

di progettualità turistica

Germana Citarella 
La narrazione spettacolarizzata del paesaggio sonoro.

Da Giuseppe Chiari a Philip K. Dick e oltre

Francesco Michi

Musica di paesaggi sonori. Enunciazione, risignificazione, comunicazione

Carlotta Sillano

Camminare per ascoltare. Partiture invisibili del territorio abitato

Elisabetta Senesi

Il paesaggio sonoro in relazione. Suono, movimento e immagini per stimolare complessità percettiva Angela Calia

Groove Fields. Understanding the Dance Floor from an Art-Based Research Perspective

Sebastian Mattbias

Il silenzio come esperienza trasformativa. L'importanza del silenzio nella meditazione e in ambito professionale

Sebastiano Caroni

Progettare il silenzio. Una lettura acustica dell'ex villaggio sanatoriale 125 Morelli a Sondalo

Martino Mocchi

Voicing One's Will. Theatre as Audio-Visual Hypotyposis of the Poetic

Michael Groneberg

Music and Clowning in Europe, 20th-21st centuries

Anna Stoll Knecht

Il paesaggio sonoro nella composizione musicale. Un percorso bibliografico

Stefano Alessandretti

\#exploreART: il labirinto di A. Pomodoro e i bambini. Un progetto di fruizione condivisa con percorsi sensoriali partecipati Alessandra De Nicola, Franca Zuccoli 


\section{OTHER EXPLORATIONS}

Il rumore lontano. Intervista a Lorena Rocca

a cura di Martino Mocchi

Re Cervo. Intervista a Antonella Astolfi

a cura di Krizia Bonaudo e Demis Quadri

Centovalli-Centoricordi. Intervista a Oliviero Giovannoni a cura di Krizia Bonaudo e Demis Quadri

Alla ricerca di un metodo: Open Space Technology 



\title{
Teatro di suoni per l'attaccamento ai luoghi. Uno sguardo geografico
}

\author{
Lorena Rocca \\ Università degli Studi di Padova; Dipartimento DFA, SUPSI
}

DoI: https://doi.org/10.7358/gn-2021-001-roc1

\begin{abstract}
Body is our access key to places: the medium that allow us to possess, belong, and identify ourselves in places. Body remembers places, mediating the twoway relationship between abstract and physical, social and mental, individual and collective perspectives. Body expresses different cultural images of societies: going through social space and making the collective experience possible. Each body is indeed a "place" in which social and political constructions take shape. The paper considers body as the starting and the ending point of the reflection, presenting a circular path for reconstructing the relationship with places through sounds. The research questions shall be as follows: when body experiences the space of theater, which geographies come to life? how does sound affect this process, developing the sense of attachment to places? why is this important - for both individuals and places?
\end{abstract}

Keywords: corporeality, place, place attachment, sound, theater.

Parole chiave: corporeità, luogo, attaccamento al luogo, suono, teatro.

\section{INTRODUZIONE}

Se, come afferma Harvey (2002), il corpo costituisce un possibile nuovo luogo teorico da cui partire, le forme teatrali diventano vie inesplorate in cui prendono forma nuovi mondi.

E, infatti, con il corpo che accediamo ai luoghi e attraverso di esso li 
possediamo, li apparteniamo, ci identifichiamo. Il corpo ha memoria dei luoghi in una sorta di dialogo aperto con essi, agisce nello spazio e, come insegna Lefebvre (1976), media la dimensione sociale e mentale, quella individuale e collettiva, secondo un rapporto biunivoco tra l'astratto e il fisico. Nel corpo si proiettano differenti immagini culturali delle società: esperiamo lo spazio sociale e, al tempo stesso, diamo senso all'esperienza sociale. Questo processo di reciprocità è inconsapevole, ma se ci pensiamo ogni corpo, in base alla posizione che occupa, è il "luogo" in cui si concentrano le costruzioni sociali e politiche.

Ricordando Foucault «il corpo è il punto zero del mondo; laddove le vie e gli spazi si incrociano, il corpo non è da nessuna parte: è al centro del mondo questo piccolo nucleo utopico a partire dal quale sogno, parlo, procedo, immagino, percepisco le cose al loro posto e anche le nego attraverso il potere infinito delle utopie che immagino. Il mio corpo è come la Città del Sole, non ha luogo, ma è da lui che nascono e si irradiano tutti i luoghi possibili, reali o utopici» $(2010,4)$.

Nel presente contributo il punto di partenza e di arrivo è quindi il corpo, in un viaggio circolare in cui si cerca di ricostruire un rapporto coevolutivo con i luoghi attraverso i suoni, nel tentativo di focalizzare l'attenzione sui seguenti aspetti: quando il corpo si trova all'interno degli spazi del teatro, quali geografie prendono vita? come il suono si configura in esse? come si sviluppa il senso di attaccamento al luogo e perché questo è importante - per sé e per i luoghi?

\section{Geografie SONORE}

I luoghi portano al loro interno dei suoni, li amplificano, li nascondono, abitano nella memoria delle persone. In particolare, un suono, che è proprio di un paesaggio, si sedimenta in esso, si cristallizza diventa un soundmark territoriale, parte di quel paesaggio grazie al valore attributo ad esso dalla comunità che vi abita. Scelsi (2010) sosteneva che i suoni possono manifestarsi una sola volta, ma i luoghi portano le sonorità con sé all'infinito. I rumori sono sempre esistiti ancor prima dei suoni prodotti dall'uomo. Da questo contenitore informe, le diverse comunità hanno estratto dei singoli suoni, separandoli. Li hanno promossi in modo più o meno inconsapevole a suoni identitari, in grado di differenziare una comunità dall'altra. Questi soundmarks territoriali si assimilano quindi nel tessuto di una società e, attraverso la memoria, si cristallizzano 
nel tempo assumendo senso e diventando memoria collettiva (Appadurai 1986) (Fig. 1).

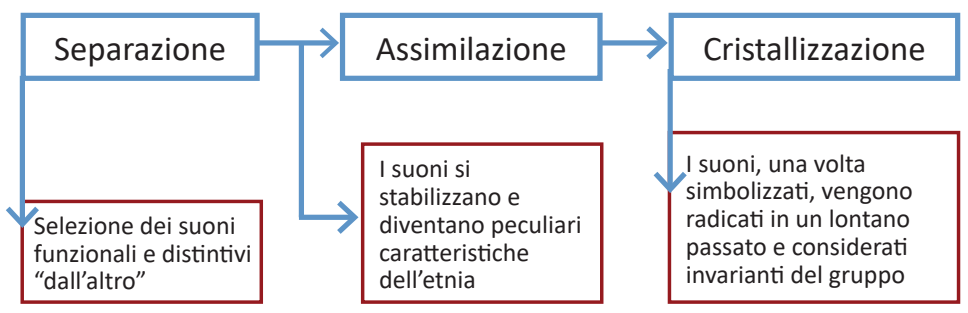

Figura 1. - Il processo di fissazione identitaria.

Fonte: elaborazione dell'autore da Remotti (1996; 2010).

\section{I SUONI COME SE...}

Continuando a ricercare le connessioni e le reciprocità, se il nostro corpo si rapporta ai luoghi in un legame coevolutivo con essi e se i suoni, cristallizzati in una memoria collettiva, diventano la base della costruzione delle identità personali, il teatro si snoda come se fosse uno spazio sul quale, attraverso i nostri corpi, sostenere e promuovere forme di attaccamento ai luoghi.

Per comprendere questo gioco di immaginazione la riflessione parte dallo spazio. L'etimologia del termine rimanda al verbo "patēre", che significa estendersi largamente, ma anche essere aperto, visibile, manifesto, raggiungibile, chiaro. Lo spazio è, infatti, ciò che è immediatamente davanti ai nostri occhi e che può essere colto prima di ogni astrazione.

Nel tempo, questa accezione ha avuto molteplici declinazioni: il vero trionfo dello spazio assoluto newtoniano vede lo spazio «indipendente dalla materia, realtà matematica, sistema di riferimento assoluto, identico, immobile, infinto» (Vagaggini 1978). Con l'approccio fenomenologico ed esistenzialista si apre una nuova strada al modo di concepire lo spazio come una costruzione del pensiero sulla base delle esperienze attraverso il corpo e i sensi. Alla luce di ciò, l'uomo è caratterizzato dall'essere-nel-mondo, in quanto la persona esistente non può mai essere pensata senza il mondo: "L'uomo non è limitato dalla superficie del suo presunto corpo. Se io sto qui, in quanto uomo, sto qui a condizione che nel contempo sto già là accanto alla finestra, cioè fuori per strada e in questa città; in breve, sono in un mondo» (Heidegger 2000, 33). 
È dunque chiaro il passaggio dall'approccio posizionale dello spazio dato dall'oggettiva distanza geometrica tra le cose, indipendentemente dal punto di vista del soggetto - allo spazio situazionale - in cui le attribuzioni di distanza o direzione sono fatte da un soggetto "in situazione".

Le forme del teatro pongono il soggetto in situazione all'interno di un spazio, che diventa luogo proprio grazie e per mezzo dei dettagli che la percezione di ciascuno coglie oltre ai ricordi e alle idee che da esso scaturiscono. Ciò contribuisce a creare dei "geogrammi” (immagini mentali) dalle realtà materiali (Raffestin 2012). Il rapporto tra "geostuttura" (lo spazio) e "geogrammi" non è lineare, essendo questi ultimi il risultato di un processo di produzione mentale, che ha origine dalla percezione anche sonora, a sua volta mediata da linguaggi differenti, selezionata e influenzata prima dal sistema sensoriale dell'individuo e poi da quello dei valori, dalle credenze, dalle sue tradizioni ${ }^{1}$.

\section{Oltre lo spettatore, i luoghi}

L'idea dell'uomo come spettatore e attore del mondo riporta a Eugenio Turri, che ne "Il paesaggio come teatro" sottolinea «uno scambio interattivo [...] tra uomo che guarda e uomo che agisce, tra attore e spettatore, tra uomo protagonista di cultura e di natura [dove] il paesaggio si pone come interfaccia tra il fare e il vedere quello che si fa, tra il guardare-rappresentare e l'agire, tra l'agire e il ri-guardare» $(1998,24)$. Turri segna il passaggio dal guardare all'agire, Gambino lo riprende e lo enfatizza: «se il paesaggio è teatro, non è tuttavia un teatro dato, con le sue scene fisse e i suoi fondali immobili, dove soltanto gli attori e gli spettatori possono cambiare; l'autorappresentazione, che consente agli attori locali di prendere le distanze dalle vicende rappresentate diventando spettatori di se stessi, ricostruisce continuamente il teatro stesso o almeno il significato che esso assume per chi partecipa in vario modo all'azione teatrale» $(2003,8)$.

${ }^{1}$ Il rapporto tra finzione/simulazione e geografia è al centro del contributo di Marcello Tanca (2019). Nelle sue riflessioni, l'autore disegna un quadro dei piani e dei dispositivi di territorializzazione che operano all'interno della "geografia della fiction". Il teatro, nella sua territorializzazione configurativa (Tanca 2019, 12), diventa a tutti gli effetti un experimentum mundi e viene letto, anche attraverso numerosi casi di studio, in modo geografico, con rigore metodologico, allo scopo di vedere qualcosa che altrimenti non saremmo in grado di vedere o di ascoltare. 
A partire da queste suggestioni, è chiaro come il teatro e l'aspetto peculiare delle sue sonorità contribuiscano a dar forma alle immagini e al processo di appropriazione e di attaccamento al reale, maturando e sviluppando il senso del luogo. Percezione e azione sono assolutamente interdipendenti: non c'è l'apprensione del reale senza l'aiuto di un'immagine e viceversa. In tal senso, il luogo offre un "centro esistenziale" al quale le persone si legano emotivamente e psicologicamente, con una forza che dipende dall'investimento emotivo di chi ne è coinvolto proprio per il fatto che i luoghi e le identità si costruiscono e costituiscono mutualmente (Convery et al 2012). Il luogo è quindi un complesso di processi attraverso i quali gli individui e i gruppi definiscono loro stessi e per questo prendono la forma di fenomeno e di esperienza. In particolare, la significazione del luogo si dimostra un fenomeno complesso, multivalente e dinamico, che coinvolge diversi processi generativi che permettono al luogo di cambiare il significato che gli viene attribuito, a seconda dell'esperienza e dei soggetti (Lukermann 1989; Relph 1976).

I luoghi hanno una storia, appaiono e divengono, con l'arrivo di nuovi elementi e la sparizione di altri più vecchi, ma anche evolvono in relazione al cambiamento delle persone che li vivono esattamente come uno spettacolo teatrale che accompagna lo spettatore all'interno della narrazione e che lo spinge a maturare il senso del luogo ovvero un rapporto intimo radicato nelle persone e non sempre consapevole ${ }^{2}$.

Il tempo è un fattore favorevole ma non indispensabile perché si crei questo legame, infatti è possibile provare attaccamento per luoghi anche

${ }^{2}$ La definizione in ambito scientifico di senso del luogo risulta sfaccettata. Graham et al. (2009) lo definiscono quale genius loci, ossia il carattere del luogo, dato dall'interazione fenomenologica tra luogo e identità. Relph (1989) e Tuan (1974) hanno enfatizzato le modalità con cui gli abitanti si appropriano di un luogo attraverso il corpo, i sensi, le percezioni e le emozioni. Con il termine "senso del luogo" essi hanno considerato sia la percezione sensoriale ottenuta attraverso sia la vista, l'udito, il gusto, l'olfatto e il tatto, sia il sentimento emotivo del luogo (Tuan 1976). Nei confronti di luoghi importanti con i quali si è creato questo sentimento, Tuan evidenzia la nascita di un rapporto di profonda affezione scaturita tra luogo fisico e sentimento umano chiamata "topofilia" (1976b). Questo forte sentimento di affetto (filia) per un luogo (topos) porta a un senso di salute e benessere e diventa parte della personalità umana. In genere siamo legati a un luogo attraverso il nostro profondo bisogno psicologico di sicurezza, stabilità, appartenenza: essere spiazzati, fuori luogo, privi di luogo, senza radici è una condizione negativa, del tutto innaturale, spesso insostenibile. La topofilia può prendere molte forme e variare in intensità e qualità di sentimento. Può essere un apprezzamento passeggero, un piacere fisico, che riporta ad esempio a elementi familiari anche semplicemente attraverso il ricordo passato (Tuan 1976). 
solo visti o di cui si è sentito parlare. Con l'aumento della familiarità verso un luogo cala l'interesse nei confronti dello stesso, poiché agli occhi di chi lo vive quotidianamente diviene un'abitudine. Il tempo ha quindi una duplice azione sul legame con il luogo: mentre lo rafforza, ne rallenta la percezione, a tal punto da rendere inudibili i suoni della quotidianità.

Non sono solo i sentimenti positivi ad accompagnare il senso del luogo. Può infatti incutere timore un palcoscenico buio, in cui l'assenza di dettagli visivi o sonori ricorda immagini paurose, o la maestosità di certi fenomeni naturali, di alcuni luoghi sacri o di particolari opere d'arte che appaiono come manifestazioni di qualcosa di sublime e durevole, che esiste di per sé stesso e trascende la vita.

Anche attraverso le forme teatrali è possibile far nascere e scaturire topofobia, topofilia, senso di attaccamento ai luoghi proprio per la capacità del teatro di eccitare, convincere, stimolare o semplicemente trasmettere sensazioni. In questo modo le forme del teatro possono aumentare l'attrattiva dei luoghi facendoli apparire desiderabili sia semplicemente per come sono, sia per i messaggi e le emozioni che ci trasmettono oppure, e questo è molto più importante, per i significati e simboli racchiusi in esso (Lando 2005, 22).

\section{Il DistaCCO E L'ATTACCAMENTO AL LUOGO ATTRAVERSO IL TEATRO DEI SUONI}

Ancora Tuan (1979) sottolinea che per maturare il senso del luogo nelle sue diverse forme è necessario un distacco fra il soggetto e il luogo: una distanza che permette al soggetto di attribuire in modo consapevole a quel luogo dei significati precisi lasciandosi coinvolgere. Ma il senso del luogo si sviluppa attraverso il place attachment, che implica il desiderare di rimanere vicini all'oggetto. Il teatro, anche attraverso le sue sonorità, crea questi giochi di vicinanza/lontananza proprio nel già citato legame spettatore/attore/percezione. Le dialettiche che contraddistinguono l'esperienza dell'attaccamento a un luogo, attraverso le forme del teatro, sono ad esempio quella movimento-pausa e quella dentro-fuori (Manzo and Devine-Wrigh 2014). Il movimento e il riposo nelle forme del teatro e nelle sue sonorità possono essere vissuti individualmente, ambientalmente, socialmente e culturalmente. L'attaccamento al luogo possiede due dimensioni (Hidalgo and Hernàndez 2001): una "fisica" e una "sociale". La prima riguarda il senso di attaccamento, che nasce dalla 
relazione con le caratteristiche fisiche del luogo; la seconda fa riferimento al legame che si insatura con le persone che fanno parte del luogo e che lo abitano (Williams et al. 1992).

Per sincronizzarsi con l'ambiente fisico, sociale e culturale attraverso le azioni, il corpo - o "body subject", riprendendo l'espressione del pensatore fenomenologico Merleau-Ponty (Manzo and Devine-Wrigh 2014) - si pone al centro del rapporto tra teatro, luoghi, attaccamento ai luoghi, prendendo vita in un place ballet, un insieme di movimenti in uno spazio-palcoscenico (anche territorialmente diffuso) che permettono di dare un significato e renderlo uno spazio interpersonale.

\section{DECALOGO DI POLARITÀ NEL TENTATIVO DI FORMULARE UNA SINTESI}

Il passaggio dallo spazio al luogo implica una relazione differente rispetto al soggetto che lo percepisce: mentre il luogo prevede infatti un rapporto di interiorità, lo spazio favorisce una relazione di esteriorità (Relph 1976). «Lo spazio si pensa, i luoghi si abitano. Lo spazio si attraversa, nei luoghi si sosta. Lo spazio è astratto, il luogo è concreto. [...] Il luogo è qualcosa che ha a che fare con la memoria, le emozioni e il desiderio, [...] una trama intessuta di rapporti» (Pozzi 2015, 15).

Il so-stare, il saper stare consapevolmente nei luoghi, è una sfida che le forme del teatro raccolgono e che l'immaginazione geografica rilancia immettendola in quella rete di comunicazione che è il mondo. La geografia, quale disciplina connettiva che seziona orizzontalmente molti dei fatti che le altre scienze approfondiscono verticalmente, crea un equilibrio tra ciò che rassicura (la scienza) e ciò che inquieta (l'arte) (Dematteis 2021, 19).

Dal balcone cognitivo della geografia si sono quindi messe in gioco delle dimensioni co-evolutive tra il luogo e il corpo, volgendo l'immaginazione ai luoghi, al senso del luogo all'attaccamento ai luoghi, con l'idea che tra le persone e i luoghi si creano delle connessioni di significato che mirano a soddisfare i bisogni umani (Relph 1976). A emergere sono delle dimensioni che potremmo declinare sostituendo alternativamente i diversi connettivi logici. L'amplissimo spettro delle opportunità fornirebbero infinite combinazioni in grado di fornire sfumature e significati dall'interno della forza poetica della geografia, che fa emergere dal fondo oscuro della terra nuovi mondi che prima non esistevano proprio grazie a un sapere al tempo stesso immaginativo e ripetitivo, rivoluzionario e 


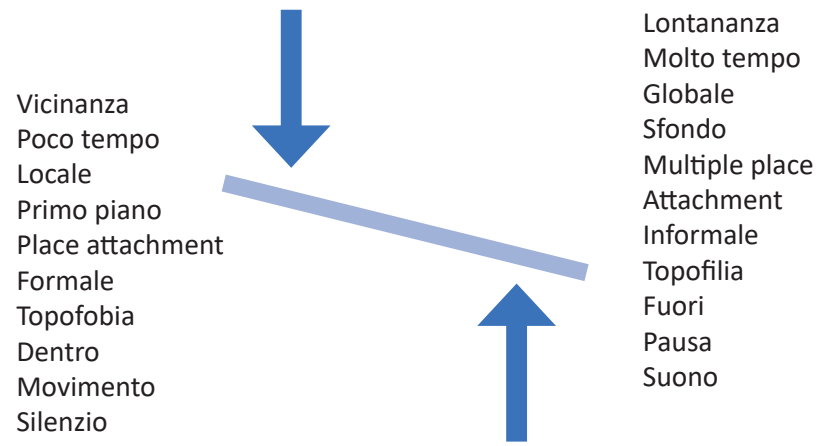

Figura 2. - Sinomini e contrari di una poetica della geografia.

Fonte: elaborazione dell'autore.

conservatore (Dematteis 2021).

Le polarità riassunte dallo schema seguente (Fig. 2) delineano percorsi di co-evoluzione tra nodi che si attraggono e respingono, grazie e per effetto delle forme del teatro di suoni. La (1) vicinanza-lontananza dal luogo è il primo tra questi. Il teatro di suoni la ricrea giocando sul (2) molto tempo-poco tempo, facendo leva sui meccanismi che le neuroscienze hanno evidenziato essere una competenza del nostro cervello. Ovvero: del suono percettivamente inseguiamo le forme, cogliendo (3) localmente le specificità che ci rimandano inevitabilmente al globale in cui il suono si colloca, e intuendo la quadridimensionalità (4) primo piano-sfondo. Come visto, attraverso le molteplici combinazioni, è possibile promuovere (5) un attaccamento a un luogo, ma anche a molti luoghi diversi, utilizzando contesti (6) formali e informali. L'appartenenza è infatti un «sentimento attivo di legame, che implica attaccamento (emozionale), e quindi sviluppa una lealtà a qualcosa cui si appartiene» (Gasparini 2000). Questo significa che quando l'individuo si impegna ad appartenere a qualcosa, lo fa perché grazie a questa appartenenza rafforza la sua identità e dà continuità alla propria esistenza e a quella del gruppo di cui fa parte. Sulla base del significato e delle esperienze mediate dalle forme teatrali, si può maturare un sentimento di benessere (7) -topofilia - proprio in una sorta di traccia curativa, oppure di sradicamento e alienazione - topofobia.

È attraverso il nostro corpo che "abitano" i suoni, gli odori, le luci di alcuni punti fissi nello spazio da cui quotidianamente partiamo e ai quali facciamo ritorno. Questo spazio risponde a un bisogno di sicurezza 
e integrazione (Galimberti 1987) e il legame spaziale che si costruisce permette di rafforzare l'identità personale. Attraverso il corpo noi abitiamo le forme del teatro. Abitare ha la stessa etimologia di abitudine, che nel significato originario designa la nostra costituzione corporea, e per estensione connota le nostre abitudini, quel che ci è abituale, ossia ciò che siamo soliti fare in una danza (8) tra il dentro e il fuori (9), il movimento la pausa. Il mio abito (babitus), ovvero il mio modo di essere, la mia predisposizione d'animo (Heidegger 2000) ${ }^{3}$.

Chiude il decalogo di sintesi la dicotomia (10) suono-silenzio: silenzio quale punto zero per l'ascolto che contribuisce a delineare le forme del teatro di suoni e al tempo stesso dei paesaggi (Turri, 2004). Attraverso suoni e silenzi sia nell'attore che nello spettatore si crea un subbuglio: il corpo registra più dati del consueto, esce dalla zona di confort e immagazzina ciò di cui non era consapevole. Per questo si rende necessario educare la memoria: il troppo non aiuta, ma offusca e confonde. Onfray in modo provocatorio sottolinea: «di un viaggio non dovrebbero restare che tre o quattro segni forse cinque o sei non di più. Tanti quanti in effetti, sono i punti cardinali necessari all'orientamento» $(2010,51)$. Forse anche la nostra sintesi dovrebbe puntare a questo per offrire, anche nelle forme del teatro di suoni, dei punti di orientamento stabili.

\section{Conclusioni}

Come sottolineato, le persone di tutte le età sentono il bisogno di sviluppare un senso del luogo per comprendere il proprio posto nel mondo (Tuan 1974). Gli studi hanno rivelato inoltre che l'attaccamento a un luogo porta a un impegno verso la comunità di appartenenza e a un coinvolgimento e interessamento nei suoi confronti. Come scrivono Brown e Perkins, l'attaccamento ai luoghi «comprende i legami positivi, talvolta presenti senza la consapevolezza, che si sono sviluppati nel tempo dalle relazioni affettive, comportamentali e cognitive tra gli individui, i gruppi e il loro ambiente socio-fisico. Questi legami provvedono a dare una cornice per gli aspetti individuali e comuni dell'identità personale» (1992,

${ }^{3}$ Come mette in evidenza Iori (1996), il dentro ("sentirsi a casa”) non sempre deve essere considerato come qualcosa di protettivo e rassicurante, così come il fuori non deve essere considerato inospitale e minaccioso. Viceversa, l'esterno può venir concepito come appetibile (perché in esso prende corpo il divertimento), e l'interno come noia e solitudine. 
284).

L'identità e l'attaccamento ai luoghi possono guidare quindi le reazioni ai luoghi e incidere su vari atteggiamenti, preferenze e comportamenti riguardo ai nostri viaggi, alle preferenze territoriali ma anche alla capacità di sviluppare una maggiore coscienza e responsabilità - motori per la partecipazione e il coinvolgimento anche nella gestione attiva del patrimonio territoriale. Oggi, di fronte alla globalizzazione, si sta vivendo un fenomeno di standardizzazione dei luoghi e per questo diventa importante dare ai luoghi una connotazione, una caratterizzazione esistenziale proponendo esperienze in grado di riporre i luoghi all'interno delle nostre identità, per ripristinare un movimento attivo nei loro confronti, un atteggiamento di presa in carico e di interesse per gli stessi perché... ci appartengono, fanno parte di noi e anche il teatro è un modo di percorrerli e ripercorrerli.

\section{RIFERIMENTI BIBLIOGRAFICI}

Appadurai, A., ed. 1989. The Social Life of Things. Commodities in Cultural Perspective. Cambridge: Cambridge University Press.

Brown, B. and Perkins, D. 1992. "Disruptions in Place Attachment", in Altman, I. and Low, S. Handbook of environmental psychology. New York: Plenum Press, pp. 279-304.

Convery, I., Corsane, G., Favis, P. 2012. Making Sense of Place: Multidisciplinary Perspectives. Woodbridge: Boydell.

Dematteis, G. 2021. Geografia come immaginazione. Tra piacere della scoperta e ricerca di futuri possibili. Milano: Hoepli.

Foucault, M. 2010. Eterotopia. Milano: Mimesis.

Galimberti, U. 1987. Gli equivoci dell'anima. Milano: Feltrinelli.

Gambino, R. 2003. "Progetto e conservazione del paesaggio". Ri-Vista, 9(23).

Gasparini, A. 2000. La sociologia degli spazi. Luoghi, città, società. Roma: Carocci.

Graham, B and Howard, P. eds. 2009. The Ashgate Research to Heritage and Identity. New York: Routledge.

Harvey, D. 2002. Spaces of Hope. Edimburgh: Edimburgh University Press.

Heidegger, M. 2000. Corpo e spazio. Genova: Il Melangolo.

Hidalgo, M. C and Hernandez, B. 2001. "Place Attachment: Conceptual and Empirical Questions”. Journal of Environmental Psychology, 21(3). pp. 273-81.

Iori, V. 1996. Lo spazio vissuto: luoghi educativi e soggettività. Firenze: La Nuova Italia. 
Lando, F. and Voltolina, A. eds. 2005. Atlante dei luoghi. Ipotesi per una didattica della geografia. Venezia: Cafoscarina.

Lefebvre, H. 1976. La produzione dello spazio. Milano: Moiozzi.

Lukermann, F. 1989. “The Nature of Geography: Post Hoc, Ergo Propter Hoc?”. In Entrikin, J.N. and Brunn, S.D. eds. Reflections on Richard Hartshorne's. The Nature of Geography. Association of American Geographers. pp. 53-68.

Manzo, L. C. and Devine Wright, P. 2014. Place Attachment: Advances in Theory, Methods and Applications. London-New York: Routledge.

Onfray, M. 2010. Filosofia del viaggio. Poetica della geografia. Bergamo: Ponte alle Grazie.

Pozzi, M. 2015. Sentimento dello spazio. Geografia affettiva dei luoghi. Roma: Linaria.

Raffestin, C. 2012, Il paesaggio come strumento progettuale per il territorio. http://goo. gl/ kptpNW/ (last access17/09/2017).

Relph, E. 1976. Place and placelessness. Pion.

Relph, E. 1989. "Responsive Methods, Geographical Imagination and the Study of Landscapes”. In Kobayashi, A. and Mackenzie, S. eds. Remaking Human Geography. Unwin Hyman. pp.149-163.

Remotti, F. 1996. Contro l'identità. Roma-Bari: Laterza.

Remotti, F. 2010. L'ossessione identitaria. Roma-Bari: Laterza.

Scelsi, G. 2010. Il sogno 101. Macerata: Quodlibet.

Tanca, M. 2019. Geografia e fiction. Opera, film, canzone, fumentto. Milano: Franco Angeli.

Tuan, Y. 1974. Topophilia. Prentice-Hall.

Tuan, Y. 1976. "Humanistic geography". Annals of the Association of American Geographers, 66(2). 266-276.

Tuan, Y. 1976b. "Literature, experience and environmental knowing”. In Moore, G.T. and Golledge R.G. eds. Environmental knowing. Theories, research and methods. Dowden, Hutchinson and Ross. 260-272.

Tuan, Y. 1979. "Sight and Pictures". Geographical Review, 69(4). 413-422.

Turri, E. 1998. Il paesaggio come teatro: dal territorio vissuto al territorio rappresentato. Venezia: Marsilio.

Turri, E. 2004. Il paesaggio e il silenzio. Venezia: Marsilio.

Vagaggini, V. ed. 2000. Spazio geografico e spazio sociale. Milano: Franco Angeli.

Williams, D. R. 1992. "Beyond the Commodity Metaphor: Examining Emotional and Symbolic Attachment to Place”. Leisure Science, 14(1). 29-46. 
